\title{
Optimal Dynamic Pricing for Binary Demands in Smart Grids: A Fair and Privacy-Preserving Strategy*
}

\author{
Jalal Arabneydi ${ }^{1}$ and Amir G. Aghdam ${ }^{2}$
}

\begin{abstract}
Motivated by demand-side management in smart grids, a decentralized controlled Markov chain formulation is proposed to model a homogeneous population of users with binary demands (i.e., off or on). The binary demands often arise in scheduling applications such as plug-in hybrid vehicles. Normally, an independent service operator (ISO) has a finite number of options when it comes to providing the users with electricity. The options represent various incentive means, generation resources, and price profiles. The objective of the ISO is to find optimal options in order to keep the distribution of demands close to a desired level (which varies with time, in general) by imposing the minimum price on the users. A Bellman equation is developed here to identify the globally team-optimal strategy. The proposed strategy is fair for all users and also protects the privacy of users. Moreover, its computational complexity increases linearly (rather than exponentially) with the number of users. A numerical example with 100 users is presented for peak-load management.
\end{abstract}

\section{INTRODUCTION}

The power grid is a complex multi-facet network consisting of various interconnected components such as generators, transmission lines, and consumers as well as marketing rules, etc. While the power grid plays a key role in today's quality of life, it increasingly faces serious challenges such as limited fuel-based energy resources, global warming, and the unpredictable nature of renewable energy sources. To adopt to the growing limitations of the future world, the traditional power grid needs to evolve to a smarter network with more efficient interactions between its components. To this end, there has been a significant surge of interest in research and investment on smart grids in recent years [1].

One of the main challenges in a smart grid is to regulate the grid in the face of different types of volatility such as the uncertainty of renewable energy or the continuous variation of daily load pattern. One of the most common and effective means of addressing this challenge is demandside management, where (shiftable and deferrable) demands are intelligently re-distributed to maintain the aggregate load close to a desired load pattern.

Several models have been proposed in the literature for demand-side management. In [2], three different classifications are introduced for these models. The first one is de-

\footnotetext{
* This work has been supported in part by the Natural Sciences and Engineering Research Council of Canada (NSERC) under Grant RGPIN262127-17, and in part by Concordia University under Horizon Postdoctoral Fellowship.

${ }^{1}$ Jalal Arabneydi and ${ }^{2}$ Amir G. Aghdam are with the Department of Electrical and Computer Engineering, Concordia University, 1455 de Maisonneuve Blvd, Montreal, QC, Canada. Email:jalal.arabneydi@mail.mcgill.ca and Email:aghdam@ece.concordia.ca
}

terministic versus stochastic, where stochastic models prove more realistic as they can capture the uncertainty of the grid. The second one is individual-based versus collaborative, where the collaborative models are more complex as they take into account the effect of each demand's decision on other demands. The third one is real-time pricing versus dayahead pricing, where day-ahead pricing structure, in addition to the current pricing, considers future pricing as well. For more details on models for demand-side management, the interested reader is referred to surveys [2]-[4] and references therein.

One of the important requirements in a smart grid is the privacy of information. This means that the status of each user should not be shared with others; consequently, only decentralized strategies are admissible in smart grids. On the other hand, it is conceptually difficult to find optimal decentralized strategies because different users have different perception of the system as their information is not necessarily the same. To address this challenge, the common practice in the literature is to first find the centralized solution and then implement it in a distributed manner by using consensus-like algorithms under some convex assumptions. In this type of approach, only a weighted average of the states of other users is available to each user. See for more details [5]-[7] on static models and [8], [9] on dynamic models.

In this paper, we propose a controlled Markov chain model for demand-side management for the case where users have a binary demand/no-demand state. This model falls into the class of stochastic, collaborative, and day-ahead pricing models. In general, binary demands emerge in various applications such as plug-in electric vehicles [10], [11], appliances scheduling [12], and pool pumps control [13]. In contrast to the papers cited in the previous paragrapgh, the dynamics, price functions and load pattern trajectory in this paper are described in discrete space (rather than continuous space). Each user has access only to its local state (private information) and the empirical distribution of demands (shared information called mean-field). In general, the computational complexity of problems with a non-classical information structure described above is NEXP [14]. Inspired by meanfield teams approach [15]-[17], we develop a Bellman equation to identify the globally team-optimal solution. It is shown that the computational complexity of the proposed solution increases linearly (rather than exponentially) with the number of users.

This paper is organized as follows. In Section II] the problem is formulated and the main challenges of finding the optimal solution are outlined. In Section III] a Bellman equa- 
tion is derived to identify the optimal solution. In Section IV, the efficiency of the proposed solution is demonstrated by numerical example and in Section $\mathrm{V}$, the paper is concluded.

\section{A. Notation}

$\mathbb{N}$ and $\mathbb{R}$ refer to natural numbers and real numbers, respectively. Given $k \in \mathbb{N}, \mathbb{N}_{k}$ denotes the finite set $\{1, \ldots, k\}$ and $x_{1: k}$ denotes vector $\operatorname{vec}\left(x_{1}, \ldots, x_{k}\right)$. The probability, expectation, and indicator functions of an event are respectively denoted by $\mathbb{P}(\cdot), \mathbb{E}[\cdot]$, and $\mathbb{1}(\cdot)$. For any $n \in \mathbb{N}$ and $p \in[0,1]$, Binopdf $(\cdot, p, n)$ denotes the binomial probability distribution of $n$ trails with success probability $p$.

\section{PROBLEM FORMULATION}

Consider an Independent Service Operator (ISO) regulating the power grid by managing the demand side. Let $n \in \mathbb{N}$ denote the number of users (consumers) in the grid. At every time instant, each user requests power (makes a demand) with probability $p \in(0,1)$. The demand processes of users are assumed to be mutually independent. It is to be noted that ISO does not necessarily serve (deliver) each demand immediately. In such a case, the corresponding user is not allowed to make a new demand until after the existing one is delivered by ISO. Denote by $x_{t}^{i} \in \mathcal{X}:=\{0,1\}$ the state of user $i \in \mathbb{N}_{n}$ at time $t \in \mathbb{N}$, where $x_{t}^{i}=1$ implies user $i$ has a demand at time $t$. Let $m_{t} \in \mathcal{M}:=\left\{0, \frac{1}{n}, \frac{2}{n}, \ldots, 1\right\}$ denote the empirical distribution of demands at time $t$, i.e.,

$$
m_{t}=\frac{1}{n} \sum_{i=1}^{n} \mathbb{1}\left(x_{t}^{i}=1\right) .
$$

For ease of reference, the empirical distribution of demands will hereafter be called mean-field.

\section{A. Model}

Suppose ISO has $k \in \mathbb{N}$ options to provide power to users, where each option has its unique price, participation, and delivery rate. The options may represent different price profiles, incentives, and generation resources. Define the following for every option $u \in \mathbb{N}_{k}$.

1) Participation rate is the probability according to which a user is incentivized to delay its demand at any time instant $t \in \mathbb{N}$. The ISO offers monetary discounts to users to incentivize them to delay their demands. Denote the participation rate $\oint^{1}$ by $\alpha(u) \in[0,1]$, and assume that it is independent of the demands. Hence, the probability of a demand being made by a participant user is $(1-\alpha(u)) p \leq p$.

2) Delivery rate is the probability according to which the demand of a user is delivered at any time $t \in \mathbb{N}$. Since ISO often uses multiple sources of energy with different time responses and capacities, it is possible that the rate of delivery changes based on the type of power generation source. Denote the delivery rate by $q\left(u, m_{t}\right) \in(0,1]$. Since

\footnotetext{
${ }^{1}$ In practice, the participation rate $\alpha(u)$ may depend on the monetary discount and the monetary discount may indirectly depend on $m_{t}$. As a result, the participation rate may be a function of $m_{t}$, and hence be denoted as $\alpha\left(u, m_{t}\right)$. For simplicity of presentation, this indirect dependence is ignored in this paper; however, our results hold for such case as well.
}

the delivery rate may decrease as the the number of demands increases, we use term $m_{t}$ to denote this dependence.

3) Reserve price is the price that a user with no demand (i.e., $x=0$ ) pays for reliable energy (i.e., for the maximum power guaranteed by the ISO). At any time instant $t \in \mathbb{N}$, ISO reserves energy for the next time instant to reduce the chances of a power outage (e.g., in case of an upcoming unexpectedly high demand). Each user must pay this price even if it does not use the electricity. Denote the reserve price by $c_{r}\left(u, 1-m_{t}\right) \in \mathbb{R}_{\geq 0}{ }^{2}$

4) Demand price is the price that a user with demand (i.e., $x=1$ ) pays for energy. This includes the price of energy as well as the price of the reserved capacity of the next time instant. It is to be noted that users with demand can use an option different from users with no demand. This flexibility enables ISO to manage its resources more efficiently. Denote the demand price by $\left.c_{d}\left(u, m_{t}\right) \in \mathbb{R}_{\geq 0}\right]^{3}$

Remark 1. Notice that $\alpha(u)=1$ and $q\left(u, m_{t}\right)=1$ mean full participation and instant delivery, respectively. When the reserve price $c_{r}\left(u, 1-m_{t}\right)$ and demand price $c_{d}\left(u, m_{t}\right)$ are independent of $m_{t}$, the price is said to be flat.

Let $u_{t}^{i} \in \mathbb{N}_{k}$ denote the option assigned to user $i \in \mathbb{N}_{n}$ at time $t \in \mathbb{N}$. Then, given $m_{t} \in \mathcal{M}$, the transition probability matrix of user $i$ at time $t$ can be written as follows:

$\mathbb{P}\left(x_{t+1}^{i} \mid x_{t}^{i}, u_{t}^{i}, m_{t}\right)=\left[\begin{array}{cc}1-\left(1-\alpha\left(u_{t}^{i}\right)\right) p & \left(1-\alpha\left(u_{t}^{i}\right)\right) p \\ q\left(u_{t}^{i}, m_{t}\right) & 1-q\left(u_{t}^{i}, m_{t}\right)\end{array}\right]$.

In addition, the price that user $i$ pays at time $t$ can be expressed as:

$$
\begin{aligned}
c\left(x_{t}^{i}, u_{t}^{i}, m_{t}\right)=\mathbb{1}\left(x_{t}^{i}=0\right) & c_{r}\left(u_{t}^{i}, 1-m_{t}\right) \\
& +\mathbb{1}\left(x_{t}^{i}=1\right) c_{d}\left(u_{t}^{i}, m_{t}\right) .
\end{aligned}
$$

\section{B. Strategy}

Any admissible strategy employed by the ISO must satisfy the following two conditions:

- They must be "fair" in the sense that no user is privileged or discriminated in the grid.

- They must be designed in such a way that the "privacy" of users is protected so that no third-party or ISO can know the status of each user.

Inspired by the above features, we consider the following control law:

$$
u_{t}^{i}=g_{t}\left(x_{t}^{i}, m_{1: t}\right),
$$

where $g_{t}: \mathcal{X} \times \mathcal{M}^{t} \rightarrow \mathbb{N}_{k}, t \in \mathbb{N}$. The collection of control laws $g_{t}$ is called the strategy of system, i.e., $g:=$ $\left\{g_{1}, g_{2}, \ldots\right\}$. This strategy is fair because if two users have the same state, their control actions will be identical. In addition, under this strategy, the state of user $i$, i.e. $x_{t}^{i}$, is known to neither ISO nor other users.

\footnotetext{
${ }^{2}$ Since the reserve price may vary based on the size of capacity (i.e. $\left.n\left(1-m_{t}\right)\right)$, we use term $\left(1-m_{t}\right)$ to denote this dependence.

${ }^{3}$ Similar to the reserve price, the demand price may vary based on the size (i.e., $n m_{t}$ ); hence, we use term $m_{t}$ to denote this dependence.
} 
Each user is equipped with a smart meter which enables the user to control its demand and communicate with the ISO and other users. There are several ways to share meanfield $m_{t}$ among users. For instance, ISO can compute the mean-field by collecting the states of users in an anonymous fashion and then broadcast the mean-field to all users. Another way is to run a proper consensus algorithm, e.g., [18], [19], in the network in order to share the mean-field among users in a distributed manner, independently of ISO.

Remark 2. When the number of users is asymptotically large, the mean-field becomes deterministic, hence predictable. In such case, there is no need to even share the mean-field [17].

\section{Optimization problem}

Let $\theta_{t} \in[0,1]$ denote the trajectory of the desired load pattern at time $t \in \mathbb{N}$, and $s_{t} \in \mathcal{S}$ denote its state space representation such that

$$
\begin{aligned}
s_{t+1} & =f\left(s_{t}\right), \\
\theta_{t} & =h\left(s_{t}\right),
\end{aligned}
$$

where $f: \mathcal{S} \rightarrow \mathcal{S}$ and $h: \mathcal{S} \rightarrow[0,1]$. The ISO provides users with initial state $s_{1}$ and functions $f$ and $h$.

The ISO ideally looks for the optimal option to keep the distribution of demands close to a desired load trajectory by imposing minimum price on users. Given discount factor $\beta \in(0,1)$, define the following cost function:

$$
J(g)=\mathbb{E}^{g}\left[\sum_{t=1}^{\infty} \beta^{t-1} \frac{1}{n}\left(\sum_{i=1}^{n} c\left(x_{t}^{i}, u_{t}^{i}, m_{t}\right)+D\left(m_{t}, \theta_{t}\right)\right)\right],
$$

where $D: \mathcal{M} \times[0,1] \rightarrow \mathbb{R}_{\geq 0}$ denotes an arbitrary distance function. Note that the above expectation function depends on the choice of strategy $g$.

Problem 1. Find the optimal strategy $g^{*}$ such that for every strategy $g$,

$$
J\left(g^{*}\right) \leq J(g)
$$

Since the information structure, given by (4), is nonclassical, every user has different information (perception) about the system and such discrepancy makes it difficult to establish cooperation among users. In general, the computational complexity of Problem 1 increases exponentially with the number of users and control horizon [14], which makes Problem 1 computationally expensive even for a relatively small number of users. This complexity is exasperated when the control horizon is infinity.

\section{MAIN RESULTS}

In this section, we present a solution to Problem 1 Given realization $m_{1: t} \in \mathcal{M}^{t}, t \in \mathbb{N}$, define reserve action $g_{t}^{r} \in \mathbb{N}_{k}$ for users with no demand as follows:

$$
g_{t}^{r}:=g_{t}\left(0, m_{1: t}\right) .
$$

Similarly, for users with demand define demand action $g_{t}^{d} \in$ $\mathbb{N}_{k}$ as:

$$
g_{t}^{d}:=g_{t}\left(1, m_{1: t}\right)
$$

Equations (4), (7), and (8) yield

$$
u_{t}^{i}=\mathbb{1}\left(x_{t}^{i}=0\right) g_{t}^{r}+\mathbb{1}\left(x_{t}^{i}=1\right) g_{t}^{d} .
$$

We show that the stochastic process $m_{1: t}$ evolves in a Markovian manner under the reserve and demand actions. This implies there is no loss of optimality in dropping $m_{1: t-1}$.

Theorem 1. For any $m_{1: t} \in \mathcal{M}^{t}, g_{1: t}^{r} \in\left(\mathbb{N}_{k}\right)^{t}$, and $g_{1: t}^{d} \in$ $\left(\mathbb{N}_{k}\right)^{t}, t \in \mathbb{N}$, the following equality holds:

$$
\mathbb{P}\left(m_{t+1} \mid m_{1: t}, g_{1: t}^{r}, g_{1: t}^{d}\right)=\mathbb{P}\left(m_{t+1} \mid m_{t}, g_{t}^{r}, g_{t}^{d}\right)
$$

Proof. From (1),

$$
n \cdot m_{t+1}=\sum_{i=1}^{n} \mathbb{1}\left(x_{t+1}^{i}=1\right) .
$$

Suppose every agent has access to $\left\{x_{1: t}^{1}, \ldots, x_{1: t}^{n}\right\}$. Then, $\left\{\mathbb{1}\left(x_{t+1}^{i}=1\right)\right\}_{i \in \mathbb{N}_{n}}$ are independent Bernoulli random variables with identical probability distribution according to (2), i.e., $\mathbb{P}\left(x_{t+1}^{i}=1 \mid x_{t}^{i}, u_{t}^{i}, m_{t}\right)$ where $u_{t}^{i}$ and $m_{t}$ are given by (9) and (1), respectively. Since (10) is a summation of $n$ independent random variables, its probability distribution can be expressed as the convolution of the probability distributions of these $n$ random variables [20]. In particular, $n\left(1-m_{t}\right)$ of the random variables have state zero at time $t$, i.e. $x_{t}^{i}=0$, and the remaining $n m_{t}$ of them have state 1 , i.e. $x_{t}^{i}=1$. Therefore, the following yields from 110 :

$$
\begin{aligned}
\mathbb{P}\left(n \cdot m_{t+1} \mid x_{1: t}^{1}, \ldots, x_{1: t}^{n}, g_{1: t}^{r}, g_{1: t}^{d}\right)= \\
\quad \underbrace{\mathbb{P}\left(1 \mid 0, g_{t}^{r}, m_{t}\right) * \ldots * \mathbb{P}\left(1 \mid 0, g_{t}^{r}, m_{t}\right)}_{n\left(1-m_{t}\right)} \\
* \underbrace{\mathbb{P}\left(1 \mid 1, g_{t}^{d}, m_{t}\right) * \ldots * \mathbb{P}\left(1 \mid 1, g_{t}^{d}, m_{t}\right)}_{n m_{t}} .
\end{aligned}
$$

On the one hand, the history of mean-field $m_{1: t}$ is a function of the history of joint state $x_{1: t}^{1}, \ldots, x_{1: t}^{n}$ according to (1), and on the other hand, the right-hand side of (11) depends only on $m_{t}, g_{t}^{r}$, and $g_{t}^{d}$. Thus, it follows from (11) that

$$
\mathbb{P}\left(m_{t+1} \mid m_{1: t}, g_{1: t}^{r}, g_{1: t}^{d}\right)=\mathbb{P}\left(m_{t+1} \mid m_{t}, g_{t}^{r}, g_{t}^{d}\right)
$$

Remark 3. It is to be noted that the mean-field process $m_{1: t}$ is not necessarily a controlled Markov process under joint actions $\left\{u_{1: t}^{1}, \ldots, u_{1: t}^{n}\right\}$, i.e.,

$$
\mathbb{P}\left(m_{t+1} \mid m_{1: t}, u_{1: t}^{1}, \ldots, u_{1: t}^{n}\right) \neq \mathbb{P}\left(m_{t+1} \mid m_{t}, u_{t}^{1}, \ldots, u_{t}^{n}\right) .
$$

Theorem 2. The transition probability matrix of the meanfield can be expressed in terms of binomial probability distributions as follows. Given any $\hat{m} \in \mathcal{M}$,

- for $m_{t}=0$,

$$
\mathbb{P}\left(m_{t+1}=\hat{m} \mid m_{t}, g_{t}^{r}, g_{t}^{d}\right)=\operatorname{Binopdf}\left(n \hat{m},\left(1-\alpha\left(g_{t}^{r}\right)\right) p, n\right),
$$


- for $m_{t}=1$,

$\mathbb{P}\left(m_{t+1}=\hat{m} \mid m_{t}, g_{t}^{r}, g_{t}^{d}\right)=\operatorname{Binopdf}\left(n \hat{m}, 1-q\left(g_{t}^{d}, m_{t}\right), n\right)$,

- for $m_{t} \notin\{0,1\}$,

$$
\begin{aligned}
& \mathbb{P}\left(m_{t+1}=\hat{m} \mid m_{t}, g_{t}^{r}, g_{t}^{d}\right)= \\
& \begin{aligned}
&\left(\operatorname{Binopdf}\left(\cdot,\left(1-\alpha\left(g_{t}^{r}\right)\right) p, n\left(1-m_{t}\right)\right)\right. \\
&\left.* \operatorname{Binopdf}\left(\cdot, 1-q\left(g_{t}^{d}, m_{t}\right), n m_{t}\right)\right)(n \hat{m}) .
\end{aligned}
\end{aligned}
$$

Proof. The proof follows from the interesting connection between convolution power and binomial distribution [20, chapter 7.1]. The first group of convolutions in (11) can be interpreted as the probability distribution of $n\left(1-m_{t}\right)$ Bernoulli random variables (trials) with success probability $\mathbb{P}\left(1 \mid 0, g_{t}^{r}, m_{t}\right)=\left(1-\alpha\left(g_{t}^{r}\right)\right) p$. Similarly, the second group of convolutions in (11) can be interpreted as the probability distribution of $n m_{t}$ trials with success probability $\mathbb{P}\left(1 \mid 1, g_{t}^{d}, m_{t}\right)=1-q\left(g_{t}^{d}, m_{t}\right)$.

Lemma 1. For any $m_{t} \in \mathcal{M}, s_{t} \in \mathcal{S}, g_{t}^{r} \in \mathbb{N}_{k}$, and $g_{t}^{d} \in \mathbb{N}_{k}$, $t \in \mathbb{N}$, there exists a function $\ell$ such that

$$
\ell\left(m_{t}, s_{t}, g_{t}^{r}, g_{t}^{d}\right)=\frac{1}{n}\left(\sum_{i=1}^{n} c\left(x_{t}^{i}, u_{t}^{i}, m_{t}\right)+D\left(m_{t}, \theta_{t}\right)\right) .
$$

Proof. The proof follows from (3), (5) and 9) as shown below

$$
\begin{aligned}
& \frac{1}{n}\left(\sum_{i=1}^{n} c\left(x_{t}^{i}, u_{t}^{i}, m_{t}\right)+D\left(m_{t}, \theta_{t}\right)\right) \\
& =\sum_{x \in \mathcal{X}}\left(\frac{1}{n} \sum_{i=1}^{n} \mathbb{1}\left(x_{t}^{i}=x\right) c\left(x_{t}^{i}, u_{t}^{i}, m_{t}\right)\right)+D\left(m_{t}, \theta_{t}\right) \\
& =\left(1-m_{t}\right) c_{r}\left(g_{t}^{r}, 1-m_{t}\right)+m_{t} c_{d}\left(g_{t}^{d}, m_{t}\right)+D\left(m_{t}, h\left(s_{t}\right)\right) \\
& =: \ell\left(m_{t}, s_{t}, g_{t}^{r}, g_{t}^{d}\right) .
\end{aligned}
$$

Theorem 3. For any $m \in \mathcal{M}$ and $s \in \mathcal{S}$, define the following Bellman equation

$$
\begin{aligned}
V(m, s):= & \min _{g^{r} \in \mathbb{N}_{k}, g^{d} \in \mathbb{N}_{k}}\left(\ell\left(m, s, g^{r}, g^{d}\right)+\right. \\
& \left.\beta \sum_{\hat{m} \in \mathcal{M}} \mathbb{P}\left(\hat{m} \mid m, g^{r}, g^{d}\right) V(\hat{m}, f(s))\right) .
\end{aligned}
$$

Let $\left(\mathfrak{g}^{r, *}(m, s), \mathfrak{g}^{d, *}(m, s)\right)$ denote a minimizer of $(12)$. Then, the optimal control law for Problem 1 is given by

$$
u_{t}^{i, *}=\mathbb{1}\left(x_{t}^{i}=0\right) \mathfrak{g}^{r, *}\left(m_{t}, s_{t}\right)+\mathbb{1}\left(x_{t}^{i}=1\right) \mathfrak{g}^{d, *}\left(m_{t}, s_{t}\right) .
$$

Proof. For any $T \in \mathbb{N}$, define the finite-horizon counterpart of $J(g)$, given by (6), as follows:

$$
J_{T}(g):=\mathbb{E}^{g}\left[\sum_{t=1}^{T} \beta^{t-1} \frac{1}{n}\left(\sum_{i=1}^{n} c\left(x_{t}^{i}, u_{t}^{i}, m_{t}\right)+D\left(m_{t}, \theta_{t}\right)\right)\right] .
$$

From (5) and Theorem 1

$$
\begin{aligned}
\mathbb{P}\left(m_{t+1}, s_{t+1} \mid\right. & \left.m_{1: t}, s_{1: t}, g_{1: t}^{r}, g_{1: t}^{d}\right) \\
& =\mathbb{P}\left(m_{t+1} \mid m_{t}, g_{t}^{r}, g_{t}^{d}\right) \mathbb{1}\left(s_{t+1}=f\left(s_{t}\right)\right) .
\end{aligned}
$$

Therefore, $\left(m_{1: t}, s_{1: t}\right)$ is a controlled Markov process under reserve and demand actions $\left(g_{1: t}^{r}, g_{1: t}^{d}\right)$. In addition, from Lemma 1, the per-step is a function of $\left(m_{t}, s_{t}, g_{t}^{r}, g_{t}^{d}\right)$. It is well-known in Markov decision theory [21] that in this case there exists a dynamic programming decomposition to identify a minimizer to (14), i.e., for $t \in \mathbb{N}_{T}$,

$$
\begin{aligned}
V_{t}(m, s):= & \min _{g^{r} \in \mathbb{N}_{k}, g^{d} \in \mathbb{N}_{k}}\left(\ell\left(m, s, g^{r}, g^{d}\right)\right. \\
& \left.+\sum_{\hat{m} \in \mathcal{M}} \mathbb{P}\left(\hat{m} \mid m, g^{r}, g^{d}\right) V_{t+1}(\hat{m}, f(s))\right),
\end{aligned}
$$

where $V_{T+1}(m, s):=0, \forall m \in \mathcal{M}, \forall s \in \mathcal{S}$. Using the proof technique of [21], we make the following change of variable for any $m \in \mathcal{M}, s \in \mathcal{S}$ and any $t \in \mathbb{N}_{T}$,

$$
W_{t}(m, s):=\beta^{-T+t-1} V_{T-t+2}(m, s),
$$

where $W_{1}(m, s)=\beta^{-T} V_{T+1}(m, s)=0$. Using simple algebraic manipulations, we arrive at

$$
\begin{aligned}
W_{T+1}(m, s)= & \min _{g r \in \mathbb{N}_{k}, g^{d} \in \mathbb{N}_{k}}\left(\ell\left(m, s, g^{r}, g^{d}\right)+\right. \\
& \left.\beta \sum_{\hat{m} \in \mathcal{M}} \mathbb{P}\left(\hat{m} \mid m, g^{r}, g^{d}\right) W_{T}(\hat{m}, f(s))\right) .
\end{aligned}
$$

Since the above Bellman operator is contractive [21], its solution converges to a fixed-point solution as time goes to infinity, i.e.,

$$
\lim _{T \rightarrow \infty} W_{T}=W_{\infty}=: V
$$

Since the mean-field and desired trajectory of demands are known to all users, every user can independently solve (12) and find the optimal strategy in a distributed manner ${ }^{4}$ Each user constructs its optimal action (13) based on the obtained strategy, private information $x_{t}^{i}$, mean-field $m_{t}$, and the state of the desired trajectory, i.e. $s_{t}$. As a result, each user will only need to know the mean-field rather than the local information of other users.

Remark 4. It is important to note that the cardinality of set $\mathcal{M}$ increases linearly with the number of users $n$ while the cardinality of set $\mathcal{S}$ is independent of $n$. Therefore, the computational complexity of the solution of the Bellman equation (12) increases linearly with $n$, given the transition probability matrix 5 in Theorem 2 Note also that the computational complexity of the Bellman equation (12) increases exponentially with time, in general; however, it is common practice to use value iteration to find an $\varepsilon$ optimal solution in polynomial time, where $\varepsilon$ converges to zero exponentially [22].

\footnotetext{
${ }^{4}$ There are various ways to solve the Bellman equation (12) such as value iteration and policy iteration [21].

${ }^{5}$ Binomial probability distribution function can be efficiently computed by different methods such as discrete Fourier transformation.
} 
According to the proof of Theorem 3 , the pair $\left(m_{t}, s_{t}\right)$ evolves in a Markovian manner under reserve and demand actions. Hence, the Bellman equation of Theorem 3 extends to the following case.

Corollary 1. Let the dynamics (2) and per-step costs (3) depend on the state of the desired load trajectory $s_{t}, t \in \mathbb{N}$. Then, the result of Theorem 3 still holds, and for any $m \in \mathcal{M}$ and $s \in \mathcal{S}$, the Bellman equation is given by

$$
\begin{aligned}
V(m, s):= & \min _{g^{r} \in \mathbb{N}_{k}, g^{d} \in \mathbb{N}_{k}}\left(\ell\left(m, s, g^{r}, g^{d}\right)+\right. \\
& \left.\quad \beta \sum_{\hat{m} \in \mathcal{M}} \mathbb{P}\left(\hat{m} \mid m, s, g^{r}, g^{d}\right) V(\hat{m}, f(s))\right) .
\end{aligned}
$$

\section{Numerical Results}

Motivated by peak-load management, we present a numerical example to illustrate our results.

Example 1. Denote by $T \in \mathbb{N}$ the number of equal control intervals within a full day (24 hours), and suppose that the ISO applies the control strategy at the beginning of each interval, i.e., it intervenes every $\frac{24 \times 60}{T}$ minutes. For any time $t \in \mathbb{N}$, define daily control time instants by the following modulo-operation:

$$
s_{t}:= \begin{cases}t-\left\lfloor\frac{t}{T}\right\rfloor T, & \frac{t}{T} \neq\left\lfloor\frac{t}{T}\right\rfloor, \\ T, & \frac{t}{T}=\left\lfloor\frac{t}{T}\right\rfloor .\end{cases}
$$

For example, let $T=100$ and $t=240$, then $s_{240}$ refers to the control interval 40 in day 3. From the above definition, $s_{t} \in \mathbb{N}_{T}, t \in \mathbb{N}$, evolves as follows:

$$
s_{t+1}=\mathbb{1}\left(s_{t}<T\right)\left(s_{t}+1\right)+\mathbb{1}\left(s_{t}=T\right),
$$

where the initial value $s_{1}=1$. Let $\tau_{B}<T$ and $\tau_{E}<$ $T$ denote respectively the first and last daily control time instants of the peak-load period. The desired load pattern trajectory is valley-shaped as follows:

$$
\theta_{t}= \begin{cases}0.8, & 1 \leq s_{t}<\tau_{B} \\ 0.8-0.6 \sin \left(\frac{s_{t}-\tau_{B}}{\tau_{E}-\tau_{B}} \pi\right), & \tau_{B} \leq s_{t}<\tau_{E} \\ 0.8, & \tau_{E} \leq s_{t} \leq T\end{cases}
$$

Suppose the ISO has the following three options $(k=3)$ at each time $t \in \mathbb{N} 6$

Basic option: This option, denoted by $u=1$, refers to the case where the electricity is provided by the main generators. In this option:

- There is no incentive for users to delay their demands, i.e., participation rate $\alpha(1)$ is 0 .

- The demands are served at rate $q\left(1, m_{t}\right)=0.2$.

- The per-user price may increase with respect to the number of served users. Let the price functions be $c_{r}\left(1,1-m_{t}\right)=1+\left(1-m_{t}\right)$ and $c_{d}\left(1, m_{t}\right)=1.5+$ $1.5 m_{t}$.

${ }^{6}$ The options can be extended to multiple electricity-providers with various price, incentive, and delivery rates.
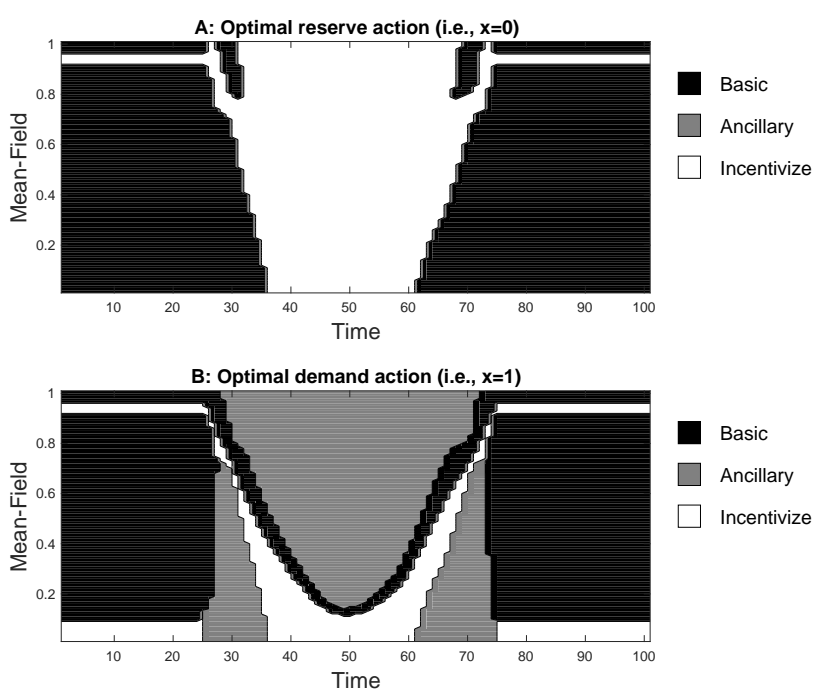

Fig. 1. The optimal strategy is shown as a function of local state $x$, meanfield $m$, and daily control time instants $s$ in Example 1. The peak-load period occurs between daily control time instants 25 and 75 . The three options (basic, ancillary, incentive-based) are depicted by three different colors.

Ancillary option: This option, denoted by $u=2$, refers to the case where ancillary (back-up) generation and reserve resources are integrated to the grid. In this option:

- There is no incentive for users to delay their demands, i.e., participation rate $\alpha(2)$ is 0 .

- The demands are served at a higher rate than that of basic option. Let $q\left(2, m_{t}\right)=0.4$.

- Let the price of electricity be flat as follows: $c_{r}(2,1-$ $\left.m_{t}\right)=2$ and $c_{d}\left(2, m_{t}\right)=3$.

Incentive-based option: This option, denoted by $u=3$, refers to the case where ISO offers monetary discounts to users for incentivizing them to cooperate with ISO. If a user has no demand at time $t$, it receives 0.05 discount unit as an incentive for not making a demand at $t+1$. If a user has a demand at time $t$, in addition to 0.05 unit discount for not sending a demand at $t+1$, it receives an extra 0.05 unit discount compared to the basic option for letting the ISO serve its current demand with more delay (lower delivery rate). In this option:

- The participation rate $\alpha(3)$ is assumed to be 0.85 .

- Let the delivery rate be given by $q\left(3, m_{t}\right)=0.15 \leq$ $q\left(1, m_{t}\right), m_{t} \in \mathcal{M}$.

- The discounted prices are assumed to be $c_{r}\left(3,1-m_{t}\right)=$ $c_{r}\left(1,1-m_{t}\right)-0.05$ and $c_{d}\left(3, m_{t}\right)=c_{d}\left(1, m_{t}\right)-0.1$.

Consider the following parameters: number of users $n=$ 100 , demand probability $p=0.8$, discount factor $\beta=0.9$, control intervals $\tau_{B}=25, \tau_{E}=75$, and scaled Euclidean distance function $D(m, \theta)=100|m-\theta|, m \in \mathcal{M}, \theta \in[0,1]$. The ISO is interested to keep the distribution of demands close to the desired trajectory by imposing minimum price on users. From Theorem 3 , the optimal strategy can be obtained by solving the Bellman equation (12). According to (13), the optimal action of user $i \in \mathbb{N}_{n}$ at time $t \in \mathbb{N}$ depends on state 
$x_{t}^{i}$, mean-field $m_{t}$, and the state of the desired trajectory $s_{t}$ (i.e., daily control time instants). The optimal strategy is displayed in Figure 1.

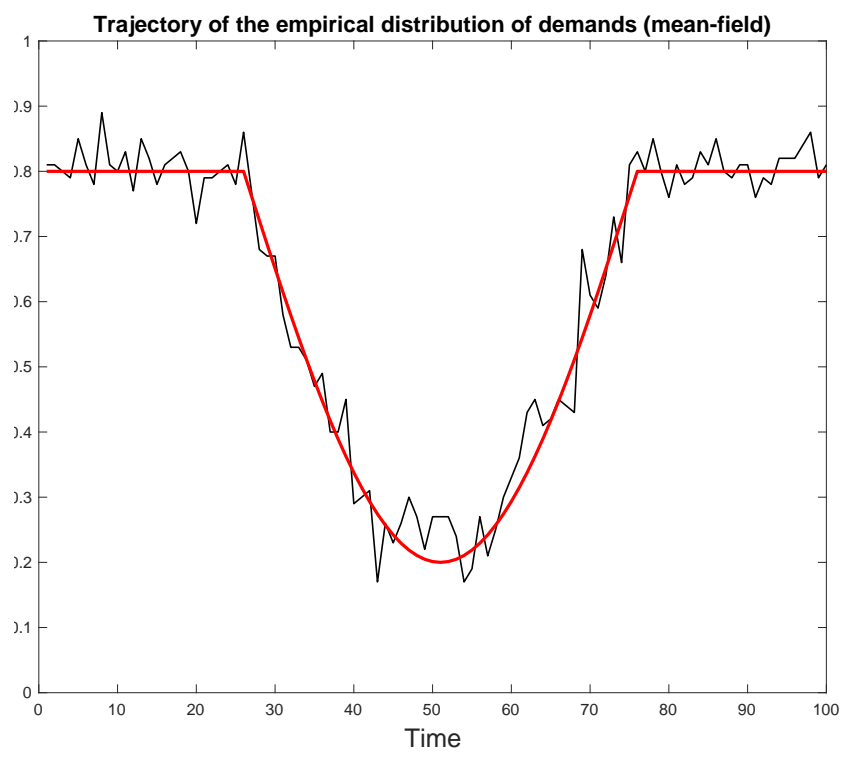

Fig. 2. The ISO aims to keep the distribution of demands (mean-field) in Example 1 close to a valley-shaped trajectory shown in red. A sample-path of the mean-field under the optimal strategy is displayed in black.

In Figure 2 a sample-path (trajectory) of mean-field (empirical distribution of demands) is depicted. Prior to the peak-load time, the number of demands is around 80 and ISO uses option 1 (basic option) for both reserve and demand actions according to Figure 11. In the peak-load time, the ISO mostly uses option 3 (incentive-based option) for users that have no demand at the moment to encourage them not to make a demand in the future. However, for users that have already requested power (i.e., have an active demand at the moment), the ISO uses option 2 (ancillary option) in combination with the other two options in order to keep the distribution of demands close to the desired trajectory with the minimum price.

\section{CONCLUSIONS}

We proposed a stochastic model for binary demands in demand-side management. Unlike many existing models, here the state and action spaces are discrete and the dynamics and cost are not necessarily smooth or convex; hence, traditional consensus-like approaches may not be as effective. The information structure is non-classical, and the computational complexity of finding a solution increases exponentially with the number of users, in general. We identified an optimal solution by the Bellman equation. Since the state space of the Bellman equation increases linearly with the number of users, the computational complexity of the proposed solution is linear with respect to the number of users. We then presented a numerical example for peak-load management to support our results.

\section{REFERENCES}

[1] X. Fang, S. Misra, G. Xue, and D. Yang, "Smart grid-the new and improved power grid: A survey," IEEE communications surveys \& tutorials, vol. 14, no. 4, pp. 944-980, 2012.

[2] A. Barbato and A. Capone, "Optimization models and methods for demand-side management of residential users: A survey," Multidisciplinary Digital Publishing Institute, Energies, vol. 7, no. 9, pp. 57875824, 2014

[3] J. S. Vardakas, N. Zorba, and C. V. Verikoukis, "A survey on demand response programs in smart grids: Pricing methods and optimization algorithms," IEEE Communications Surveys \& Tutorials, vol. 17, no. 1, pp. 152-178, 2015.

[4] R. Deng, Z. Yang, M.-Y. Chow, and J. Chen, "A survey on demand response in smart grids: Mathematical models and approaches," IEEE Transactions on Industrial Informatics, vol. 11, no. 3, pp. 570-582, 2015.

[5] P. Samadi, A.-H. Mohsenian-Rad, R. Schober, V. W. Wong, and J. Jatskevich, "Optimal real-time pricing algorithm based on utility maximization for smart grid," in Proceedings of the 1st IEEE International Conference on Smart Grid Communications, pp. 415-420, 2010.

[6] A. D. Dominguez-Garcia and C. N. Hadjicostis, "Distributed algorithms for control of demand response and distributed energy resources," in Proceedings of 50th IEEE Decision and Control and European Control Conference (CDC-ECC), pp. 27-32, 2011.

[7] K. Sakurama and M. Miura, "Communication-based decentralized demand response for smart microgrids," IEEE Transactions on Industrial Electronics, vol. 64, no. 6, pp. 5192-5202, 2017.

[8] Z. Tan, P. Yang, and A. Nehorai, "An optimal and distributed demand response strategy with electric vehicles in the smart grid," IEEE Transactions on Smart Grid, vol. 5, no. 2, pp. 861-869, 2014.

[9] T.-H. Chang, M. Alizadeh, and A. Scaglione, "Coordinated home energy management for real-time power balancing," IEEE Power and Energy Society General Meeting, pp. 1-8, 2012.

[10] N. Rotering and M. Ilic, "Optimal charge control of plug-in hybrid electric vehicles in deregulated electricity markets," IEEE Transactions on Power Systems, vol. 26, no. 3, pp. 1021-1029, 2011.

[11] J. M. Foster and M. C. Caramanis, "Optimal power market participation of plug-in electric vehicles pooled by distribution feeder," IEEE Transactions on power systems, vol. 28, no. 3, pp. 2065-2076, 2013.

[12] I. Koutsopoulos and L. Tassiulas, "Optimal control policies for power demand scheduling in the smart grid," IEEE Journal on Selected Areas in Communications, vol. 30, no. 6, pp. 1049-1060, 2012.

[13] S. P. Meyn, P. Barooah, A. Bušić, Y. Chen, and J. Ehren, "Ancillary service to the grid using intelligent deferrable loads," IEEE Transactions on Automatic Control, vol. 60, no. 11, pp. 2847-2862, 2015.

[14] D. S. Bernstein, R. Givan, N. Immerman, and S. Zilberstein, "The complexity of decentralized control of Markov decision processes," INFORMS, Mathematics of operations research, vol. 27, no. 4, pp. 819-840, Nov. 2002.

[15] J. Arabneydi, "New concepts in team theory: Mean field teams and reinforcement learning," Ph.D. dissertation, McGill University, 2016.

[16] J. Arabneydi and A. Mahajan, "Team optimal control of coupled subsystems with mean-field sharing," in Proceedings of 53rd IEEE Conference on Decision and Control, pp. 1669-1674, 2014.

[17] J. Arabneydi and A. G. Aghdam, "A certainty equivalence result in team-optimal control of mean-field coupled Markov chains," in Proceedings of the 56th IEEE Conference on Decision and Control, pp. 3125-3130, 2017.

[18] R. Olfati-Saber, E. Franco, E. Frazzoli, and J. S. Shamma, "Belief consensus and distributed hypothesis testing in sensor networks," Springer, Networked Embedded Sensing and Control, vol. 331, pp. 169-182, Jul. 2006.

[19] A. N. Bishop and A. Doucet, "Distributed nonlinear consensus in the space of probability measures," Elsevier, 19th IFAC Proceedings Volumes, vol. 47, no. 3, pp. $8662-8668,2014$.

[20] C. M. Grinstead and J. L. Snell, Introduction to probability. American Mathematical Society, 2012.

[21] P. R. Kumar and P. Varaiya, Stochastic systems: Estimation, identification, and adaptive control. SIAM (Classics in Applied Mathematics), 2015, vol. 75.

[22] D. P. Bertsekas, Dynamic programming and optimal control. Athena Scientific, 2012. 